\title{
DESENVOLVIMENTO DE SISTEMA ESTRUTURADO COM \\ INTELIGÊNCIA ARTIFICIAL PARA APOIO NO DIAGNÓSTICO \\ DE PARASITOSES INTESTINAIS
}

DEVELOPMENT OF A STRUCTURED ARTIFICIAL

INTELLIGENCE SYSTEM TO SUPPORT THE DIAGNOSIS

OF INTESTINAL PARASITOSES

Clin Biomed Res. 2020;40(3):148-153

1 Departamento de Ciências da Vida e Saúde, Universidade do Oeste de Santa Catarina. São Miguel do Oeste, SC, Brasil.

2 Departamento de Engenharia Elétrica, Centro Universitário Internacional. São Miguel do Oeste, SC, Brasil.

3 Faculdade de Medicina, Universidade de Passo Fundo. Passo Fundo, RS,Brasil.

4 Departamento de Ciências da Computação, Universidade do Oeste de Santa Catarina. São Miguel do Oeste, SC, Brasil.

Autor correspondente: Eduardo Ottobelli Chielle eduardo.chielle@unoesc.edu.br Departamento de Ciências da Vida e Saúde, Universidade do Oeste de Santa Catarina

Rua Oiapoc, 211. 89900-000, São Miguel do Oeste, $\mathrm{SC}$, Brasil.

\section{Eduardo Ottobelli Chielle ${ }^{1}$, Eliseu Luiz Kuiava², Guilherme Augusto Faust ${ }^{1}$, Ana Paula Ottobelli Chielle ${ }^{1}$, Victor Antônio Kuiava ${ }^{3}$, Roberson Junior Fernandes Alves ${ }^{4}$}

\section{RESUMO}

Introdução: Sistemas de inteligência artificial são tecnologias promissoras de assistência em saúde e diagnóstico laboratorial, que podem ser implementados como métodos de suporte para o diagnóstico de parasitoses intestinais. Este estudo objetivou desenvolver um software de IA que auxilia no diagnóstico laboratorial de parasitoses intestinais, com alta sensibilidade e especificidade.

Métodos: O software foi desenvolvido utilizando duas redes neurais, Inception e MobileNet. Primeiro imagens de ovos dos parasitas Ascaris lumbricoides, Trichiuris trichiura, Taenia sp, Hymenolepis nana, Schistosoma mansoni e larvas de Strongyloides stercoralis, foram utilizados para treinar o banco de dados. Posteriormente 2.740 imagens cedidas pelo Laboratório de Parasitologia da Universidade do Oeste de Santa Catarina foram testadas no software.

Resultados: O software apresentou sensibilidade de $82,3 \%$ (95\% intervalo de confiança (IC), 71,9\%-89,1\%) e especificidade de 95,1\% (95\% IC, 94,3\%-97,8\%) para MobileNet e sensibilidade de 72,1\% (95\% IC, 52,6\%-115\%) e especificidade de $92,1 \%$ (95\% IC, 91,7\%-97,7\%) para Inception.

Conclusão: O software apresentou resultados promissores na análise de parasitas intestinais, reforçando que, no futuro, a presença de sistemas de suporte de diagnóstico das parasitoses pode vir a se tornar mais rápido e eficiente.

Palavras-chave: Parasitas; inteligência artificial; diagnóstico

\section{ABSTRACT}

Introduction: Artificial intelligence systems are promising technologies for health care and laboratory diagnosis, which can be implemented as support methods for the diagnosis of intestinal parasitoses. This study aimed to develop an artificial intelligence software that assists the laboratory diagnosis of intestinal parasitoses with high sensitivity and specificity.

Methods: The software was developed using two neural networks, Inception and MobileNet. First, images of eggs from the parasites Ascaris lumbricoides, Trichuris trichiura, Taenia sp., Hymenolepis nana, Schistosoma mansoni and Strongyloides stercoralis larvae were used to train the database. Then, 2,740 images provided by the Parasitology Laboratory of the Universidade do Oeste de Santa Catarina were tested in the software.

Results: The software had a sensitivity of $82.3 \%$ (95\% confidence interval [Cl], $71.9 \%-89.1 \%)$ and a specificity of $95.1 \%(95 \% \mathrm{Cl}, 94.3 \%-97,8 \%)$ for MobileNet and a sensitivity of $72.1 \%(95 \% \mathrm{Cl}, 52.6 \%-115 \%)$ and a specificity of $92.1 \%(95 \% \mathrm{Cl}$, $91.7 \%-97.7 \%)$ for Inception. 
Conclusion: The software showed promising results in the analysis of intestinal parasites, reinforcing that, in the future, the presence of diagnostic support systems for parasitoses may become faster and more efficient.

Keywords: Parasites; artificial intelligence; diagnosis

\section{INTRODUÇÃO}

Os sistemas de saúde ao redor do mundo precisam enfrentar numerosos e simultâneos desafios para conseguir prover à população formas de prevenção, diagnóstico e tratamento das mais variadas doenças e condições parasitológicas. Tais desafios se apresentam em diversas esferas, e estão geralmente ligados às demandas da população, falta de eficiência sistemas de saúde e condições precárias da população1.

Isto se torna evidente, especialmente em países subdesenvolvidos e em desenvolvimento onde as parasitoses intestinais apresentam uma marcante incidência e constituem um problema de saúde pública, contribuindo para o agravamento de problemas econômicos, sociais e de saúde ${ }^{2}$. Estudos feitos no Brasil apontam uma significativa prevalência de parasitoses nas diferentes populações que habitam o país ${ }^{3,4}$. A prevalência e o tipo de parasita se apresentam bastante variados, pois sofrem influência da diversidade geográfica, etnias, culturas e costumes ${ }^{5}$. Do ponto de vista parasitológico, pessoas de baixa classe econômica e social, que vivem em áreas com más condições de higiene, estão mais suscetíveis para infecção por enteroparasitoses, de modo que a qualidade de vida, e os hábitos de higiene são fatores determinantes para o aparecimento das doenças ${ }^{6}$.

Existe uma elevada incidência de doenças parasitárias, principalmente em indivíduos que vivem em condições expostas ou que estejam em desvantagem social. Enteroparasitoses atingem indivíduos de todas as faixas etárias, causando anemia, diarreia, emagrecimento, má absorção dos nutrientes, dificuldade para aprendizado, diminuição no rendimento do trabalho e retardo no desenvolvimento físico e cognitivo ${ }^{7}$.

No entanto, os avanços tecnológicos e científicos associados ao diagnóstico destas parasitoses pararam no tempo, e atualmente não há disponível no mercado equipamentos ou softwares que auxiliem no diagnóstico mais rápido e preciso destas parasitoses. O diagnóstico em sua grande maioria, ainda se limita a observação microscópica e individual de cada amostra, tornando a prática laboratorial em parasitologia desgastante e cansativa ${ }^{8}$. Nesse contexto, o uso de técnicas computacionais estruturada com Inteligência Artificial (IA), para o diagnóstico, torna-se uma ferramenta importante que auxilia os profissionais de saúde e especialmente os pacientes, aprimorando e agilizando os diagnósticos ${ }^{9}$.
A IA tem sido alvo de muita discussão atualmente, pois consiste em um conjunto de tecnologias computacionais que são inspiradas em sistemas neurais com a finalidade de sentir, aprender, reagir e criar ações. Ao contrário do ser humano elas diferem em velocidade, autonomia e generalidade. Seu papel na sociedade tem sido ainda pouco expressivo, contudo estimativas preveem maior uso delas em poucos anos, com grande ênfase para sistemas de transportes autônomos, diagnósticos médicos, tratamentos e assistência física para pessoas mais velhas $^{10,11}$. Na área de saúde, a utilização de sistemas de IA tem obtido grande avanço, sendo vista como uma das principais áreas de atuação, podendo muito bem assistir diagnósticos e procedimentos em saúde ${ }^{10}$.

Nesse sentido, um importante segmento de atuação da IA consiste no auxílio do diagnóstico laboratorial, uma vez que o sistema de reconhecimento visual e o deep learning dos aplicativos de IA já estão bem estruturados, podendo ser de grande valia 0 desenvolvimento de sistemas que pudessem ajudar os profissionais de saúde com os diagnósticos ${ }^{10}$. Assim, este estudo objetivou desenvolver um software de IA que auxilia no diagnóstico laboratorial de parasitoses intestinais, com alta sensibilidade e especificidade, 0 qual pode ser utilizado por laboratórios, especialmente os que trabalham para o Sistema Único de Saúde (SUS), agilizando e aumentando os diagnósticos das parasitoses intestinais, tanto na distinção de normalidade e patologia.

\section{MÉTODOS}

\section{Desenvolvimento do software}

Um software de IA foi desenvolvido usando a técnica de aprendizado profundo. Este software foi estruturado em um conjunto de algoritmos que modelam abstrações de dados de alto nível usando um gráfico profundo com várias camadas de processamento, compostas por transformações lineares e não lineares. Foi estabelecido uma interface de comunicação das características das imagens de parasitas intestinais (ovos e larvas) organizadas sob uma cadeia de análise que definiu um padrão lógico de classificação de imagens com e sem estruturas parasitárias.

A linguagem de programação Python versão 3 foi adotada. Essa linguagem promove um método sistemático de programação baseado no conceito 
de software estruturado em blocos, facilitando a identificação das características no banco de dados. É uma linguagem dinâmica, interpretada, robusta, multiplataforma e multiparadigma (orientação a objetos, funcional, reflexiva e imperativa). As linguagens de programação PHP versão 7 e Javascript permitiram criar uma interface de fácil manipulação, favorecendo a implementação de botões e barras de rolagem para escolher alternativas e os comandos necessários.

O software desenvolvido foi denominado de ParasitAl (Parasites Artificial Intelligence).

\section{Desenvolvimento do banco de dados e seleção de imagens parasitológicas}

As imagens parasitológicas foram removidas de sites especializados em parasitologia clínica, com diferentes resoluções. As imagens foram analisadas pelos pesquisadores e classificadas de acordo com a estrutura e seu gênero e espécie por Farmacêutico-Bioquímico com mais de 10 anos de experiência em análises parasitológicas. Estas imagens posteriormente foram utilizadas para 0 treinamento do banco de imagens do software.

Para o desenvolvimento do banco de dados do sistema, foram utilizadas 95 imagens selecionadas, redimensionadas, recortadas e trabalhadas de modo geral, possibilitando a geração de 4.224 imagens. Ao final do processo foram obtidas 704 imagens de Ovos de Ascaris lumbricoides, 704 imagens de Trichiuris trichiura, 704 imagens de larvas de Strongyloides stercoralis, 704 imagens de ovos de Taenia sp., 704 imagens de Schistosoma mansoni e 704 imagens de Hymenolepis nana, conforme Figura 1.

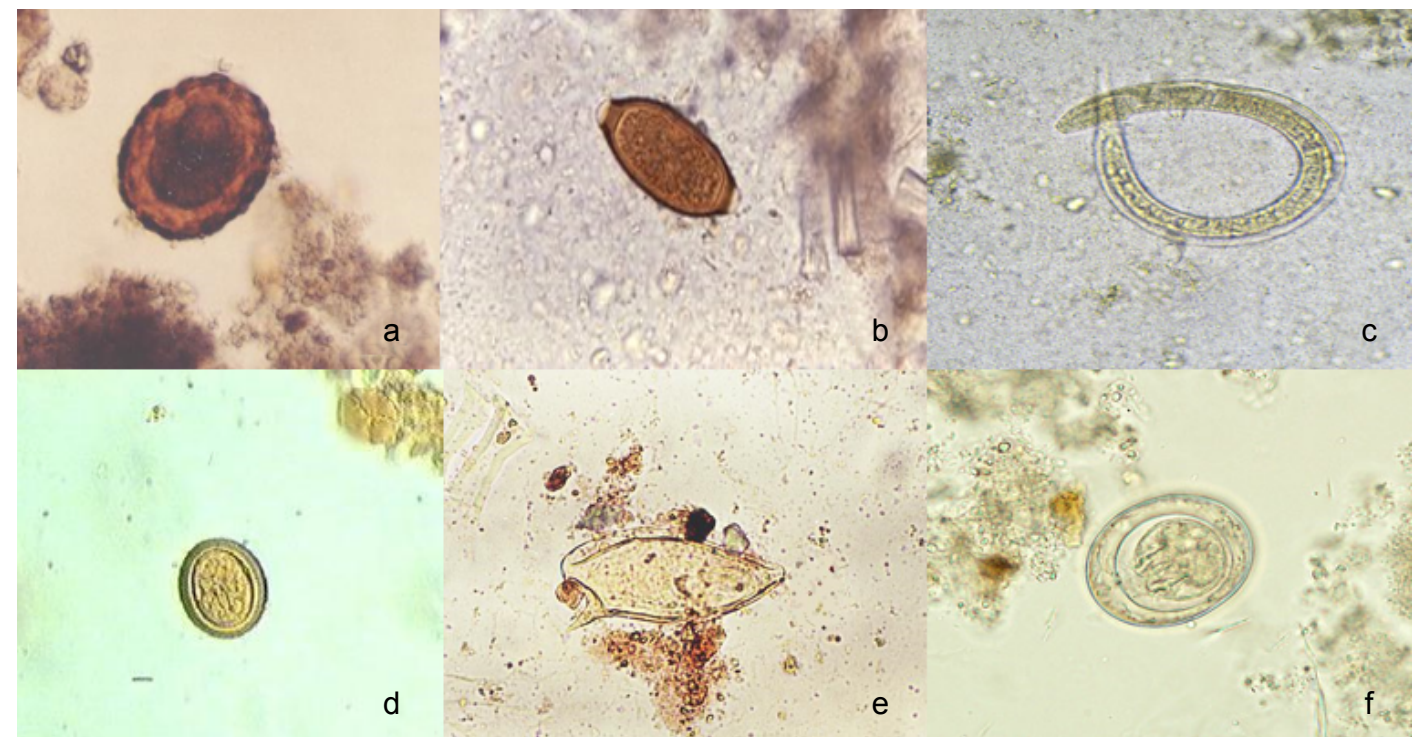

Figura 1: Modelo de imagem usado para o desenvolvimento do banco de dados de software. a: Ovos de Ascaris lumbricoides; b: Ovos de Trichuris trichiura; c: Larvas de Strongyloides stercoralis com primórdio genital evidente; d: Ovos de de Taenia sp.; e: Ovos de Schistosoma mansoni; f: Ovos de Hymenolepis nana.

Todas as imagens selecionadas foram analisadas pelo software de inteligência artificial para criar um padrão de reconhecimento. A resolução da imagem do sistema é 224x224 pixels para o MobileNet e 299x299 para o Inception. Utilizou-se análise de imagem com três canais $R, G$ e $B$.

\section{Treinamento do algoritmo de inteligência artificial}

A técnica Deep Learning com treinamento em rede neural convolucional foi aplicada através da biblioteca TensorFlow de código aberto. Dois modelos de redes neurais pré-treinados, Mobilenet versão 1.0 e Inception versão 3 , foram utilizados para treinar a classificação das imagens. Ambos os modelos foram arquitetados a partir de um conjunto de imagens de uso geral pré-analisadas, disponíveis pelo Google Inc.
O treinamento do algoritmo de inteligência artificial utilizou as 4224 imagens selecionadas e classificadas pelos pesquisadores nos modelos de rede neural pré-treinados MobileNet e Inception. Dez por cento (422) das imagens foram usadas como um conjunto de dados para a validação interna do próprio sistema, a fim de permitir o cálculo dos níveis de confiabilidade.

As configurações usadas para calibrar o MobileNet e obter o melhor desempenho foram: 1200 interações foram configuradas, com uma taxa de aprendizado de 0,001 , cujo nível de confiabilidade oferecido pelo sistema foi de $86 \%$.

Para o Inception, a melhor configuração encontrada foi uma série de interações de 5000 , com uma taxa de aprendizado de 0,1 , cujo nível de confiabilidade oferecido pelo sistema foi de $81,9 \%$. 


\section{Validação de software}

Após o treinamento do software, este foi submetido a um teste de eficiência, que ocorreu em três etapas:

Primeiro: foram selecionadas 120 imagens dos parasitas testados, que geraram 685 imagens de diferentes aumentos microscópicos de 40 e 100x (432 imagens sem estruturas parasitárias, 268 de larvas Strongyloides stercoralis, 520 de ovos de Ascaris lumbricoides, 412 ovos de Taenia sp., 540 de Schistosoma mansoni, 568 de Trichiuris trichiura e 249 de Hymenolepis nana). As lâminas e imagens foram cedidas pelo Laboratório de Parasitologia da Universidade do Oeste de Santa Catarina - São Miguel do Oeste - Brasil.

Segundo: o diagnóstico das lâminas foi reconfirmado por Farmacêutico-Bioquímico e as 685 imagens foram convertidas para o formato JPEG e inseridas no programa.
As 685 imagens fornecidas pelo Laboratório de Parasitologia, passaram por rotações de 90, 180 e 270 graus para aumentar o número do banco de dados de imagens que foram testadas pelo sistema. Assim, 2.740 imagens foram usadas para validar externamente o sistema.

Terceiro: as imagens no formato JPEG foram analisadas pelo software ParasitAl, que liberou 0 diagnóstico em um arquivo de texto que posteriormente foi tabulado no Microsoft Excel.

\section{RESULTADOS}

O software foi desenvolvido para verificar imagens de estruturas parasitárias presentes em Exames Parasitológicos de Fezes, treinado para realizar diagnósticos, diferenciando os parasitas intestinais de alimentos processados pelo trato gastrointestinal. Sua estrutura é mostrada na Figura 2.

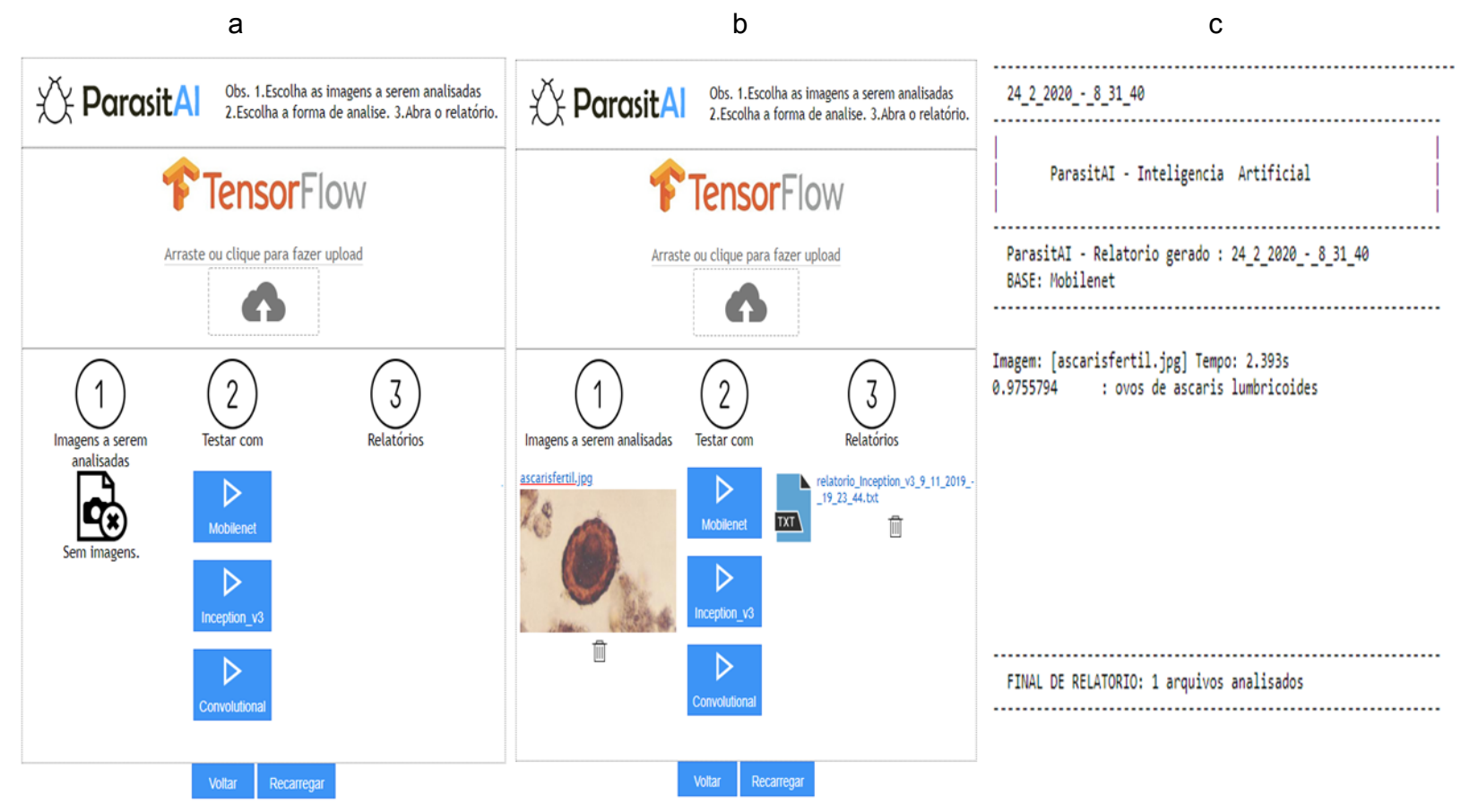

Figura 2: Estrutura funcional do ParasitAl (Parasitas Artificial Intelligence). a: Página inicial; b: Página de inserção da imagem em JPEG; c: Página com relatório de resultados.

A sensibilidade e a especificidade do software foram verificadas por meio do teste de 2.740 imagens fornecidas pelo Laboratório de Parasitologia da Universidade do Oeste de Santa Catarina (432 imagens sem estruturas parasitárias, 268 de larvas Strongyloides stercoralis, 520 de ovos de Ascaris lumbricoides, 412 ovos de Taenia sp., 440 de Schistosoma mansoni, 419 de Trichiuris trichiura e 249 de Hymenolepis nana). Os resultados globais apresentaram sensibilidade de 82,3\% [95\% intervalo de confiança (IC), 71,9\%-89,1\%] e especificidade de $95,1 \%$ (95\% IC, 94,3\%-97,8\%) para MobileNet, e sensibilidade de $72,1 \%$ (95\% IC, 52,6\%-115\%) e especificidade de $92,1 \%$ (95\% IC, 91,7\%-97,7\%) para Inception (Tabela 1). Para diferenciação entre imagens sem parasitas intestinais e com presença de parasitas o sistema obteve sensibilidade máxima de $77,6 \%$ e especificidade de $94,6 \%$. Não houve diferença estatística em sensibilidade e especificidade entre MobileNet e Inception $(p=0,74)$. 
Chielle et al.

Tabela 1: Sensibilidade e especificidade por parasita intestinal avaliado.

\begin{tabular}{lccc}
\hline Condições & MobileNet & Inception \\
\hline \multirow{2}{*}{ Ascaris lumbricoides } & Sensibilidade & 0,8824786325 & 0,8760683761 \\
Strongyloides stercoralis & Especificidade & 0,9480659026 & 0,9140401146 \\
& Sensibilidade & 0,8211538462 & 0,8307692308 \\
Taenia sp & Especificidade & 0,9903295129 & 0,9835243553 \\
\multirow{2}{*}{ Schistosoma mansoni } & Sensibilidade & 0,7384615385 & 0,5961538462 \\
& Especificidade & 0,9856733524 & 0,9885386819 \\
Trichiuris trichiura & Sensibilidade & 0,6204379562 & 0,2357043236 \\
\multirow{2}{*}{ Hymenolepis nana } & Especificidade & 0,9774355301 & 0,9881805158 \\
\multirow{2}{*}{ Global } & Sensibilidade & 0,9195205479 & 0,9332191781 \\
& Especificidade & 0,9362464183 & 0,9058022923 \\
& Sensibilidade & 0,822815534 & 0,8495145631 \\
& Especificidade & 0,9674068768 & 0,9580945559 \\
& Sensibilidade & 0,8251575931 & 0,7211805158 \\
& Especificidade & 0,9510315186 & 0,9216361032 \\
\hline
\end{tabular}

\section{DISCUSSÃO}

A incidência e prevalência de parasitoses, principalmente no que tange a população de baixa renda, ainda é um problema de saúde pública em países subdesenvolvidos ${ }^{12,13}$. Desta forma, os processos de detecção precoces e corretos diagnósticos das parasitoses dependem, dentre outros fatores, do raciocínio e experiência de profissionais de saúde. Nesse contexto, o uso de técnicas computacionais no diagnóstico estruturado com IA torna-se uma ferramenta importante que poderá ajudar os profissionais de saúde e especialmente os pacientes, melhorando e acelerando o diagnóstico de parasitoses ${ }^{10,13}$. Este trabalho propôs o desenvolvimento de um software que poderá auxiliar no diagnóstico de parasitoses intestinais.

Os resultados obtidos mostraram que o sistema ParasitAl gerou dados promissores, apresentando sensibilidade de $77,6 \%$ e especificidade de $94,6 \%$, com características inovadoras que poderão ser incorporadas em laboratórios de parasitologia, no futuro.

Alguns estudos têm demonstrado que sistemas de IA podem até superar os profissionais no diagnóstico de enfermidades específicas. Um estudo publicado em Annals of Oncology Scientific Journal, por Mar e Soyer, com participação de cientistas da França e Estados Unidos, mostrou que patologistas detectaram com precisão $86,6 \%$ de melanomas, enquanto um sistema de IA obteve acerto em $95 \%$ dos casos $^{14}$. Bringsjord et al. ${ }^{10}$ descreveram que na área da saúde, o uso de sistemas de IA tem apresentado significativos progressos, sendo assim visto como uma das principais formas de auxiliar diagnósticos.

Sistemas de apoio à decisão são softwares que se propõem a ajudar os clínicos no diagnóstico e decisões terapêuticas relacionadas ao paciente. Eles facilitam o acesso dos profissionais a dados precisos na literatura científica, possibilidades propedêuticas, estabelecimento de diagnóstico diferencial, além de destacar características específicas em novos padrões clínicos ${ }^{13}$.

O sistema de leitura e identificação facilita a leitura e diagnóstico de parasitoses intestinais, permitindo aos laboratórios e profissionais diagnosticar as mesmas estruturas à distância, como em cidades afastadas de centros metropolitanos, vilas e regiões ribeirinhas que não contam com profissionais capacitados para o diagnóstico. Pode-se rapidamente preparar a amostra no microscópio, fotografar e realizar um diagnóstico prévio, e/ou mais tarde enviar as imagens para lugares distantes, como grandes centros especializados que apresentam tecnologia e profissionais capazes de confirmar o diagnóstico ${ }^{15,16}$.

Esse tipo de procedimento tem potencial de reduzir vários custos, como por exemplo os de transporte de material, evitando a perda de amostras, e também garantindo a realização de um diagnóstico mais rápido e assertivo ${ }^{16,17}$. Com base no banco de dados, o sistema de leitura e diagnóstico é capaz de disponibilizar recomendações mais enfáticas para cada caso de parasitoses em alguns minutos, mostrando o melhor tratamento. O sistema diferencia-se por possuir dois bancos de dados pré-treinados, que potencializa a validação dos dados, garante maior segurança de resultados e melhor validação externa.

Levando em conta que este trabalho é o resultado de um conceito preliminar, existem limitações no mesmo, como o uso de somente dois modelos de rede neural para desenvolvimento do software e captura de imagens clássicas das condições analisadas, os parasitas usados na testagem são estruturas facilmente identificáveis na rotina do EPF. Um desequilíbrio foi observado no número de imagens usadas para cada condição patológica analisada. A ferramenta teria potencial para inserção sistemática em rotina laboratorial futuramente, após ser aperfeiçoada e associada a outros sistemas de leitura de lâminas como captação automática de imagens (scanner). 
Formas que demandam maior treinamento para identificação correta de enteroparasitos, gerando constantemente maiores dúvidas tanto durante o aprendizado quando no laboratório clínico, são os cistos de protozoários intestinais, os quais não foram inclusos neste estudo inicial, sendo necessário um incremento da capacidade analítica do sistema para a diferenciação de detalhes estruturais. Entretanto, enfatizamos que é um método prático, barato e efetivo e que os próximos passos deverão ser o aperfeiçoamento da capacidade analítica e maior treinamento do sistema, para melhorar em sua qualidade e consequentemente a especificidade e sensibilidade.

Destacamos o fato de que é improvável que uma máquina substitua inteiramente profissionais humanos, embora esse sistema IA pode auxiliar o diagnóstico de parasitoses intestinais ${ }^{11,18}$. Além do mais, esse sistema de interpretação e diagnóstico com base em IAé capaz de ler, interpretar, diferenciar e diagnosticar imagens de ovos e larvas de parasitas intestinais, tornando o diagnóstico mais preciso, eficiente e rápido, especialmente em lugares onde há uma frágil e escassa estrutura de diagnóstico.

Sistemas de IA vêm desempenhando um papel mais significativo em diversas áreas, em especial na área da saúde, que possuem um grande potencial no auxílio a assistência e diagnóstico. Constituem modos de complementar as fraquezas estruturais do sistema de saúde, como por exemplo a indisponibilidade de profissionais e limitações geográficas.

O sistema desenvolvido obteve resultados preliminares de pré-treinamento e validação importantes e indicaram que a ferramenta tem potencial de uso para triagem diagnóstica e no futuro com um incremento no banco de dados visando aumentar a diversidade dos parasitos deverá ser realizada para aperfeiçoar o sistema.

\section{Conflitos de Interesses}

Os autores declaram não ter conflitos de interesse.

\section{REFERÊNCIAS}

1. Saldiva PHN, Veras M. Gastos públicos com saúde: breve histórico, situação atual e perspectivas futuras. Estud av. 2018;32(92):47-61.

2. Silva CG, Santos HA. Ocorrência de parasitoses intestinais da área de abrangência do Centro de Saúde Cícero Idelfonso da Regional Oeste da Prefeitura Municipal de Belo Horizonte, Minas Gerais. Bioterra. 2001;1(1):1-11.

3. Andrade EC, Leite ICG, Vieira MT, Abramo C, Tibiriçá SHC, Silva PL. Prevalência de parasitoses intestinais em comunidade quilombola no Município de Bias Fortes, Estado de Minas Gerais, Brasil, 2008. Epidemiol Serv Saúde. 2011;20(3):337-44.

4. Ely LS, Engroff P, Lopes GT, Werlang M, Gomes I, De Carli GA. Prevalência de enteroparasitos em idosos. Rev bras geriatr gerontol. 2011;14(4):637-46.

5. Marquez AS, Marquez AS, Hasenack BS, Trapp EH, Guilherme RL.

Prevalência de enteroparasitoses em crianças de um bairro de baixa renda de Londrina - Paraná. UNOPAR Cient Cienc Biol Saude. 2002;4(1):55-9.

6. Damazio SM, Lima MS, Soares AP, Souza MAA. Intestinal parasites in a quilombola community of the
Northern State of Espírito Santo, Brazil. Rev Inst Med trop S Paulo. 2013;55(3):179-83.

7. Prado MS, Barreto ML, Strina $A$, Faria JAS, Nobre AA, Jesus SR. Prevalência e intensidade de infecção por parasitas intestinais em crianças na idade escolar na cidade de Salvador (Bahia, Brasil). Rev Soc Bra Med Trop. 2001;34(1):99-101.

8. Santos PHS, Barros RCS, Gomes KVG, Nery AA, Casotti CA. Prevalência de parasitoses intestinais e fatores associados em idosos. Rev Bras Geriatr Gerontol. 2017;20(2):244-54.

9. Gebrim LH. A detecção precoce do câncer de mama no Brasil. Cad Saude Publica. 2016;32(5):1-2.

10. Bringsjord S, Bello P, Ferrucci D. Creativity, the Turing Test, and the (better) Lovelace Test. Minds Mach. 2001;11:3-27. 11. Sugi M, Murakami H, Yoshida K. Projection of future changes in the frequency of intense tropical cyclones. Clim Dyn. 2017;49:619-32.

12. Frei F, Juncansen $C$, Ribeiro-Paes JT. Levantamento epidemiológico das parasitoses intestinais: viés analítico decorrente do tratamento profilático. Cad Saude Publica. 2008;24(12):2919-25.

13. Panch T, Szolovits $P$, Atun R. Artificial intelligence, machine learning and health systems. J Glob Health. 2018;8(2):30-7.

14. Mar VJ, Soyer PH. Artificial intelligence for melanoma diagnosis: how can we deliver on the promise? Ann Oncol. 2018;30(12):e1-e3.

15. Krizhevsky A, Sutskever I, Hinton GE. ImageNet classification with deep convolutional neural networks. Commun ACM. 2017;60(6):84-90.

16. Gunkel DJ. Comunicação e inteligência artificial: novos desafios e oportunidades para a pesquisa em comunicação. Galáxia. 2017;(34):5-19.

17. Atun R. Transitioning health systems for multimorbidity. Lancet. 2015;386(9995):721-2.

18. Badawi O, Brennan T, Celi LA, Feng M, Ghassemi M, Ippolito A, et al. Making big data useful for health care: a summary of the inaugural mit critical data conference. JMIR Med Inform. 2014;2(2):1-11. 